\title{
Effect of tickborne illnesses on women
}

By Tedra Smith, DNP, CPNP-PC, CNE; Elizabeth Coleman, MSN, CRNP, CPNP-PC; and Aimee Chism Holland, DNP, WHNP-BC, FNP-C, RD, FAANP

In this article, the authors use two case studies to describe the assessment of patients with potential tickborne illness. Criteria for the diagnosis and current recommendations for treatment of Rocky Mountain spotted fever and Lyme disease are reviewed. Patient education for prevention of tickborne illnesses is discussed. Readers are provided with the link to a video with a step-by-step simulation on tick removal.

KEY WORDS: tickborne illnesses, Rocky Mountain spotted fever, Lyme disease, tick removal

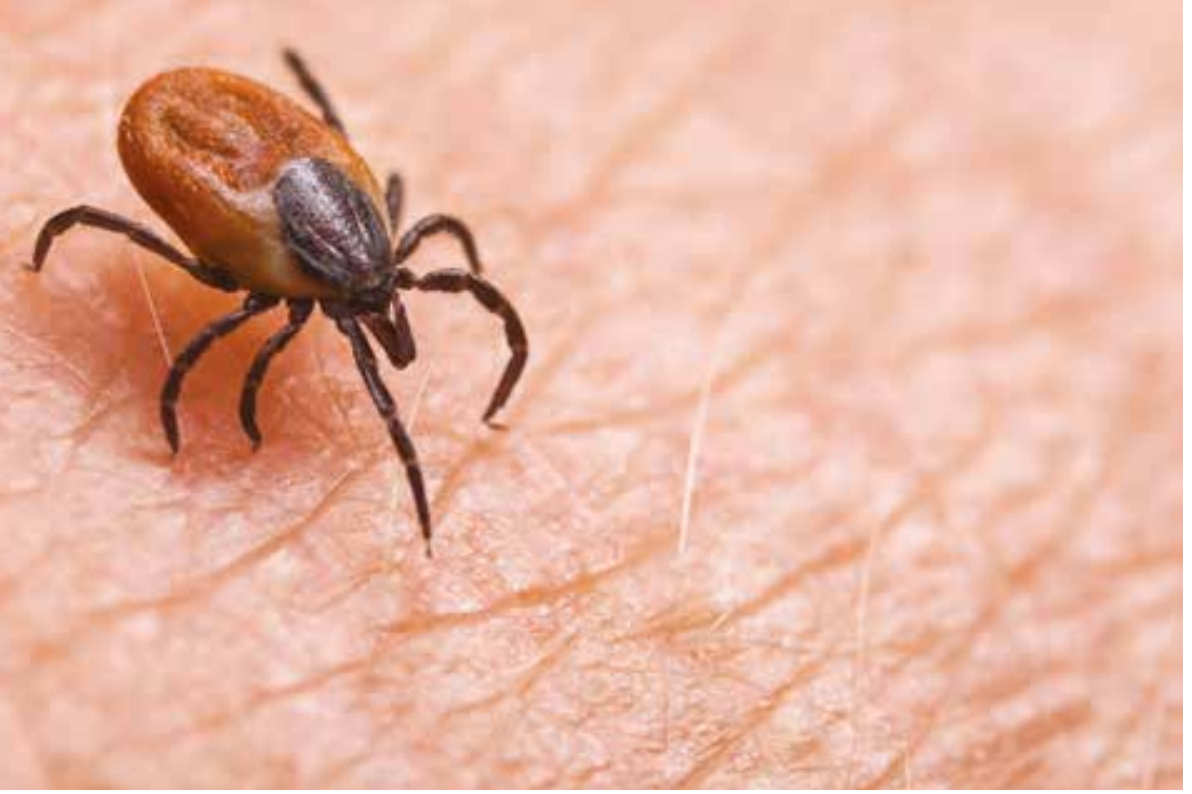

\section{Case study} presentation-Rocky Mountain spotted fever

A 29-year-old woman, ML, comes into the clinic reporting that over the past week she has felt dizzy, fatigued, and has had body aches, including a headache. She states that "my temperature has been over 101.0 ${ }^{\circ}$." She also reports having a rash that has spread over most of her body. ML denies any known sick contacts at her job and says that it is only she and her daughter at home.

\section{What other information would be helpful in making the diagnosis?}

$\mathrm{ML}$ mentions that she has been working outside for her most recent job. She started work 2 weeks ago, in June, for a landscaping company and has been clearing an area with heavy brush surrounded by tall grass. Her daughter has been helping her at work, as she is on summer vacation and $M L$ does not always have a babysitter. ML's daughter has not had any recent health issues and is doing well. ML reports that she, as well, is usually healthy and does not take any daily medications. ML has not been in close contact with anyone who was sick or with similar symptoms. ML notes that she has been wearing short 
sleeve tops and shorts while working outside. Even though she never saw an insect or felt a painful bite, she thinks she may have been bitten on her leg where the rash started. ML has never had a similar rash before, although her other symptoms remind her of when she had the flu. $M L$ also recalls that she had the fever before the rash started. Initially, the rash began on her arms and legs and then spread to her abdomen and chest. The rash is not itchy or painful. ML has not used any medications or herbal remedies to try to alleviate her symptoms. ML also feels dizzy at times and has some muscle pain. She reports a mild case of diarrhea this morning. ML has not travelled out of state recently or out of the country in the past year. She and her daughter are both up to date on their vaccinations.

On further discussion, the nurse practitioner (NP) learns there is a possibility that $\mathrm{ML}$ is pregnant. She typically has a regular menstrual cycle but is approximately 2 weeks late for menstruation at this point. She and her husband had been trying for a second child for some time, and had almost forgotten about it, but remembered once the NP asked about her menstrual cycle.

\section{What should the NP include in the physical examination?}

The NP should carefully examine the rash, noting the color, whether there is blanching, any apparent pattern or tenderness over the area, and any exudate. The rash associated with Rocky Mountain spotted fever (RMSF) typically begins as small, blanching, erythematous macules (Photo 1) on the extremities, sometimes including the palms and soles, and can spread to the trunk. ${ }^{1}$ The RMSF rash can then become petechial. ${ }^{1}$ The NP should also include an abdominal exam, noting

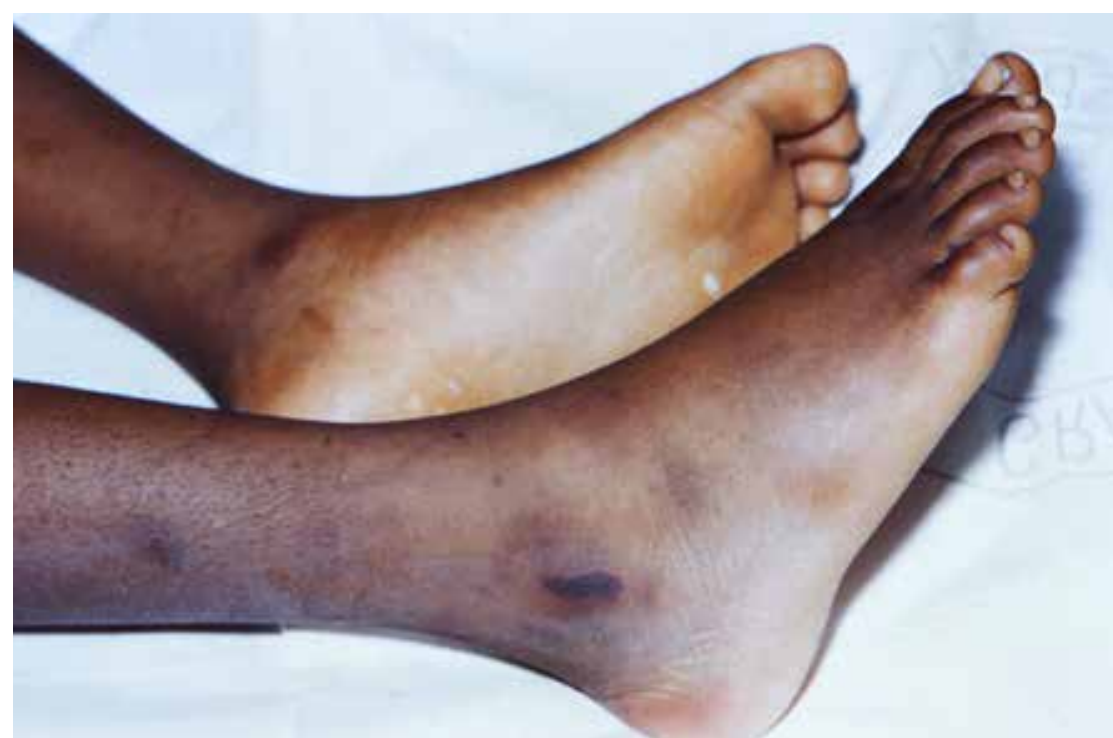

Photo 1. Rocky Mountain spotted fever.

The feet and lower legs of a hospital patient with macular eruptions due to Rocky Mountain spotted fever caused by Rickettsia rickettsii transmitted through an infected tick. Centers for Disease Control and Prevention.

any tenderness or masses in the abdominal area. A neurologic exam should be included, as this system is commonly affected in RMSF. ${ }^{1}$ The NP should pay special attention to any deficits seen on exam. During the HEENT [head, eyes, ears, nose, and throat] exam, the NP should pay careful attention to the eyes, looking for conjunctival injection, and should ask about light intolerance.

\section{What are the examination findings for this patient?}

The physical examination revealed a diffuse, blanching maculopapular rash found on the arms, legs, trunk, and back. Bowel sounds were hyperactive, and some generalized tenderness was noted during palpation of the abdomen. The neurologic exam was normal, although the patient reported some dizziness during the examination. No physical evidence of a tick or tick body part was found on the patient.

\section{What are the differential diagnoses based on the previous information?}

Differential diagnoses include influenza, toxic shock syndrome, Rocky Mountain spotted fever, Lyme disease, and meningococcemia.

\section{What is the probable diagnosis based on the symptoms and physical examination?}

RMSF is the most likely diagnosis based on the history, symptoms, and physical examination of the patient.

\section{What diagnostic studies are needed?}

The diagnosis of RMSF is usually based on clinical signs and symptoms and history suggesting possible tick exposure. Antibiotic therapy should be started immediately if RMSF is suspected and not delayed while waiting for laboratory confirmation. ${ }^{2}$ The two most commonly used tests for RMSF include indirect fluorescent antibody (IFA) testing on a skin biopsy from the area of a skin lesion or an IFA test from blood drawn within the first week of the onset of symptoms and again 2 to 3 weeks later. ${ }^{1,2}$ An increase of the IFA titer should be fourfold. ${ }^{2}$ The patient might also have thrombocytopenia, hyponatremia, and leukocytosis. ${ }^{1,2}$ The NP will need to order a pregnancy test. A urine test initially would be helpful in determining medication management for this condition. 
appropriate attire when Outdoors,

which includes wearing long pants

tucked into socks and long-sleeved shirts, using tick repellents, taking a shower immediately after being outdoors, and inspecting the skin for the presence of ticks after outdoor exposure.

\section{RMSF pathophysiology}

RMSF is caused by Rickettsia rickettsii, a Gram-negative coccobacillus, typically transmitted by the American dog tick (Dermacentor variabilis), the Rocky Mountain wood tick ( $D$. andersoni), and the brown dog tick (Rhipicephalus sanguineus). ${ }^{1}$ Unfortunately, the cases of RMSF have been increasing. In 2000, there were less than two identified cases per million people, and in 2010, there were over six cases per million people. ${ }^{1}$ The rickettsiae enter the bloodstream and then the endothelial cells of capillaries, where the rickettsiae multiply and then cause cell death. ${ }^{1}$ The incubation period of RMSF is 2 to 14 days. ${ }^{1}$ After this time period, patients typically show signs and symptoms of fever, chills, malaise, headaches, and muscle pain. Complications are associated with neurologic sequelae, specifically behavioral and cognitive disturbances. ${ }^{1}$ Patients might have issues with hyperactivity, problems concentrating, memory problems, residual seizures, and weakness. ${ }^{1}$ The mortality rate of RMSF is less than $0.5 \%{ }^{1}$

\section{What should be included in the management plan?}

According to the Centers for Disease Control and Prevention, doxycycline $100 \mathrm{mg}$ twice daily by mouth for 7 days is the recommended antibiotic to treat RMSF. ${ }^{2,3}$ The length of treatment may need to be extended if the patient remains febrile until 2 days after the patient becomes afebrile. ${ }^{2}$ The patient should follow up if symptoms do not subside or if any new symptoms occur, especially any neurologic symptoms. If ML's pregnancy test comes back positive, the NP will need to make a clinical decision based on the severity of the patient's illness whether to prescribe doxycycline or chloramphenicol because a tetracycline antibiotic could cause permanent teeth discoloration and enamel hypoplasia in a fetus. ${ }^{1,2}$ The provider should consider a consult with a maternal fetal medicine specialist as well if the pregnancy test is positive.

\section{Case study presentation-Lyme disease}

RC, a 31-year-old woman, comes to the clinic with the chief complaint of "a leg rash that looks like a bull's eye."
She had been out hiking at a state park last week. A few days after the hike, she noticed what appeared to be a tiny, black insect on the back of her leg. She removed the insect, but the next day she noticed a small rash that looked like a red ring on her leg. The rash has been slowly increasing in size each day. $\mathrm{RC}$ reports that the rash feels slightly warm but does not itch. She thought it was ringworm based on an internet search, so she purchased fungal cream over the counter and applied it daily, but it does not seem to be helping. RC reports feeling more fatigued than usual and reports taking medication for a fever the day before coming to the clinic.

\section{What other information would be helpful to making the diagnosis?}

The NP will need to assess for any other associated symptoms, such as headache, nausea, fever, or neurologic deficits. In the early localized stage of Lyme disease, the patient may have only minor constitutional symptoms. The patient may not realize or remember possible associated symptoms until asked.

\section{What should the NP include in the physical examination?} The NP should perform a thorough skin assessment, searching for an attached tick, any additional lesions, and regional lymphadenopathy. It is also important to inspect for a retained tick limb embedded in the patient's skin. The NP should assess the rash for blanching, diameter, tenderness, and warmth. Typically, in Lyme disease, the rash will appear as a "bull's eye" pattern, also known as erythema migrans (EM) rash. ${ }^{4}$ The EM rash usually is warm but not tender. Many cases of Lyme disease, however, do not present with EM, or sometimes they can have rashes that 
resemble other diseases. EM should be at least $5 \mathrm{~cm}$ in diameter to meet the criteria for Lyme disease. ${ }^{4}$

\section{What are the examination findings for this patient?}

The physical examination revealed an erythematous, blanchable circular rash with a target-like center on the posterior side of the left lower leg and left inguinal lymphadenopathy. There were not any visible signs of a tick or tick body part. A review of her vital signs noted a temperature of $100.4^{\circ} \mathrm{F}$. The exam was otherwise benign.

\section{What are the differential diagnoses based on the case presentation?}

Differential diagnoses for Lyme disease are very similar to RMSF. The signs and symptoms overlap with a number of different illnesses such as fibromyalgia, RMSF, vitamin B12 deficiency, multiple sclerosis, lead poisoning, and Lou Gehrig's disease. ${ }^{5}$

\section{What is the probable diagnosis based on the symptoms and physical examination?}

Lyme disease is the most likely diagnosis based on the history, symptoms, and physical examination of the patient.

\section{What diagnostic studies are needed?}

The NP should use the two-step method for diagnosis, the first step being an enzyme immunoassay test from a blood sample. The second step is a Western blot, performed if the first step is positive. ${ }^{4}$ However, there is some debate over whether testing is necessary if clinical presentation suggests Lyme disease. ${ }^{4}$ Given that the patient is within a childbearing age range, a pregnancy test should be completed prior to determining a management plan.

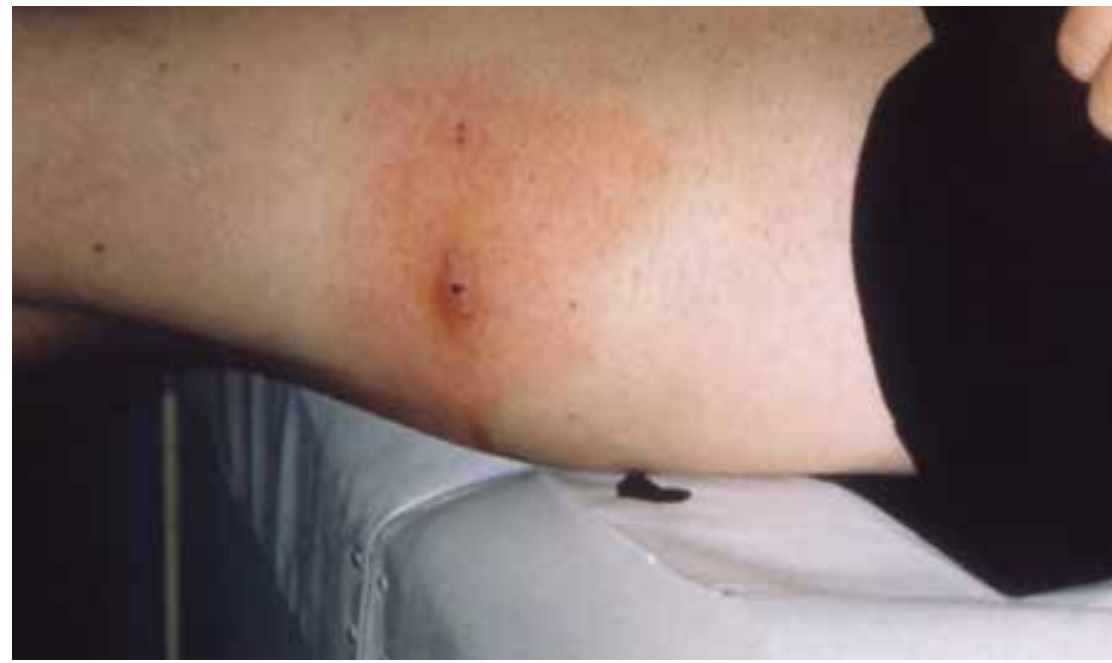

Photo 2. Lyme disease.

Lateral aspect of the left thigh of a patient presenting with what was diagnosed as Lyme disease. Note characteristic red, expanding rash called erythema migrans caused by Borrelia burgdorferi. B. burgdorferi bacteria are transmitted to humans through the bite of infected blacklegged ticks. Centers for Disease Control and Prevention.

\section{Lyme disease pathophysiology}

Lyme disease is caused by a spirochete, Borrelia burgdorferi, and is considered the most common vector-borne disease in the United States, affecting about 300,000 people each year. ${ }^{5}$ Although the overall total cases appear to be decreasing, the geographic distribution is increasing among counties in the Northeast and Midwest. The disease is usually transmitted from an infected immature black-legged tick (also known as deer tick) to humans through a bite. Typically, the tick must be attached to a person for 36 to 48 hours before the Lyme disease bacterium can be transmitted. Removal of the tick within 24 hours greatly reduces the chances of Lyme disease.

Lyme disease symptoms, which include EM, fever, chills, headache, swollen lymph nodes, and fatigue may take 3 to 30 days to appear. It is important to note that EM may present as an erythematous circular rash without the bullseye, target-like appearance (Photo 2). Late symptoms such as severe joint pain, facial palsy, heart palpitations, and neck stiffness may occur days to months after the initial tick bite. ${ }^{5}$ Lyme disease left untreated can cause nerve pain, episodes of dizziness, as well as inflammation of the brain and spinal cord. Patients should be told to report fever, rash, and flu-like symptoms up to 2 weeks after completion of the initial treatment. Post-treatment Lyme disease is a syndrome that could occur up to 6 months after treatment. Patients may experience prolonged pain, fatigue, and possibly difficulty thinking. The cause of the post-treatment syndrome is unknown, but it is believed that Lyme disease triggers an autoimmune response that is responsible for the symptoms.

\section{What should be included in the management plan?}

The complete removal of the tick itself, if found, is imperative to the effectiveness of the management plan. Readers can click this video link kaltura.com/tiny/rxirsh ${ }^{A}$ to access a presentation of a simulated stepby-step tick removal. Steps for tick removal are outlined in the Box. The International Lyme and Associated Diseases Society identified treatment guidelines for Lyme disease that should be followed. ${ }^{6}$ Doxy- 
Box. Steps for tick removal

1. Don non-latex gloves

2. Clean the affected area with a povidone iodine swab (3 times in a circular motion)

3. Use a magnifying glass to locate the head of the tick

4. Hold the tweezers close to the skin surface grasping the end (head) of the tick

5. Use the tweezers to pull upward with a steady motion (avoid jerking) to remove the tick

6. Once the tick is removed, clean the surface with an alcohol pad

7. Assess the area to ensure the entire tick was removed

8. Place the live tick in alcohol to kill it or flush it down a toilet

Video link kaltura.com/tiny/rxirsh: This video is the intellectual property of the University of Alabama at Birmingham and cannot be shared without request of a license (please contact tedraka@uab. edu). NPWH members are encouraged to share the article itself, which contains this link, with their colleagues.

cycline, $100 \mathrm{mg}$ twice daily by mouth for 10 to 21 days, is the treatment recommended for the localized or early disseminated stage of Lyme disease. Amoxicillin (500 mg bid for 14-21 days) or cefuroxime (500 $\mathrm{mg}$ tid for 14-21 days) are alternate treatment recommendations. ${ }^{5}$ If the pregnancy test comes back positive, however, the NP will need to make a clinical decision about which antibiotic to prescribe based on the severity of the patient's illness. ${ }^{6}$ The NP will most likely prescribe amoxicillin or cefuroxime if RC's pregnancy test is positive.

Patients should be reassessed at the end of 21 days. When persistent signs and symptoms are present, the patient should be evaluated for other potential causes prior to adding additional antibiotics to the regimen. ${ }^{6}$ Late disseminated stage of Lyme disease is treated with the same antibiotics, although the length of treatment is 28 days. ${ }^{7}$ Intravenous antibiotics may be needed if arthritis symptoms (persistent, inflammatory joints) do not improve with the oral regimen.

\section{Prevention of tickborne illnesses}

Preventive measures include wearing appropriate attire when outdoors, which includes wearing long pants tucked into socks and long-sleeved shirts. Preventive measures also include using tick repellents, taking a shower immediately after being outdoors, and inspecting the skin for the presence of ticks after outdoor exposure. Another preventive step is to ensure that pets, especially dogs, are treated for ticks and checked for ticks daily. Outdoor workers are at a particularly increased risk of contracting disease through ticks and other insects if they work in areas with high grass, wooded areas, and brush. ${ }^{8}$ Patients who may be at risk for tick exposure should also be advised on correct tick removal.

\section{Reflection}

It is important to include questions related to possible exposure to ticks during the history when patients present with similar history and symptom profiles as seen in the case studies. This includes asking about outdoor adventures such as hiking as well as recent travel into a wooded area. There are multiple severe complications related to tickborne illnesses, which include long-term neurologic deficits and digit loss. Preventive measures should be discussed.

Tedra Smith is Assistant Professor and Pediatric Partnership Director, Elizabeth Coleman is Instructor of Nursing, and Aimee Chism Holland is Associate Professor and DNP Program Director, all at the University of Alabama at Birmingham School of Nursing. The authors have no actual or potential conflicts of interest in relation to the contents of this article.

Acknowledgments: The authors would like to acknowledge the inspiration for this article. Dr. Holland's cousin, K. Ellis, was recently diagnosed with both Lyme disease and Rocky Mountain spotted fever after experiencing numerous complex symptoms that went undiagnosed for many years. Thankfully, a specialist has been able to provide treatment for the coexisting infections. The authors also would like to thank Mr. James Clark, an instructional design specialist at the University of Alabama at Birmingham School of Nursing, for recording the video.

\section{References}

1. Seraji-Bozorgzad N, Tselis AC. Non-Lyme tick-borne diseases: a neurological perspective. Curr Neurol Neurosci Rep. 2013;13(10):1-14.

2. Lin L, Decker CF. Rocky mountain spotted fever. Dis Mon. 2012;58(6):361-369.

3. Centers for Disease Control and Prevention. Rocky Mountain spotted fever (RMSF). Treatment. 2018. https://www.cdc.gov/rmsf/treatment/index.html.

4. Moore KS. Lyme disease: diagnosis, treatment guidelines, and controversy. J Nurse Practitioners. 2015;11(1):64-69.e2.

5. Centers for Disease Control and Prevention. Lyme disease. 2019 https://www.cdc.gov/lyme/index. html.

6. Cameron DJ, Johnson LB, Maloney EL. Evidence assessments and guideline recommendations in Lyme disease: the clinical management of known tick bites, erythema migrans rashes and persistent disease. Expert Rev Anti Infect Ther. 2014;12(9):1103-1135.

7. Schoen RT. Lyme disease: diagnosis and treatment. Curr Opin Rheumatol. 2020;32(3):247-254.

8. Kohanna F. Tick-borne illnesses. Professional Safety. 2016;61(6):83-84.

Web resource

A. kaltura.com/tiny/rxrsh 